\title{
Two problems of George Andrews on generating functions for partitions
}

\author{
Wenchang Chu
}




\title{
TWO PROBLEMS OF GEORGE ANDREWS ON GENERATING FUNCTIONS FOR PARTITIONS
}

\author{
WENCHANG CHU
}

Received 8 June, 2011

\begin{abstract}
Based on Andrews' recent work on parity in partitions, this paper will prove two partition identities proposed by Andrews (2010), simplify two generating functions into single sum expressions and extend two double series expansions of the first and second $q$-exponential functions.
\end{abstract}

2000 Mathematics Subject Classification: 11P81; 05A19; 33D15

Keywords: partition identities, generating functions, the $q$-binomial theorem

In a recent paper [1] on parity in partition identities, Andrews proposed fifteen problems. The two of them, numbered with 9 and 10 and soon proved by Yee [5], demand essentially to confirm the following identities:

$$
\begin{aligned}
& \sum_{n \geq 1}(-1)^{n} q^{n^{2}}=\sum_{i, j \geq 0} \frac{(-1)^{i} q^{(i-j)^{2}+j^{2}+i-j}}{(-q ; q)_{i}(q ; q)_{2 j-1}(q ; q)_{i-2 j+1}}, \\
& \sum_{n \geq 0}(-1)^{n} q^{n^{2}}=\sum_{i, j \geq 0} \frac{(-1)^{i} q^{(i-j)^{2}+j^{2}+j}}{(-q ; q)_{i}(q ; q)_{2 j}(q ; q)_{i-2 j}} ;
\end{aligned}
$$

where the summation index $n$ in the second equation should start from 0 instead of 1. Here we follow the standard notation of shifted factorials in base $q$ given by

$$
(x ; q)_{0}=1 \text { and }(x ; q)_{n}=(1-x)(1-q x) \cdots\left(1-q^{n-1} x\right) \text { for } n \in \mathbb{N}
$$

which admit for $|q|<1$ the two well-defined infinite product expressions

$$
(x ; q)_{\infty}=\prod_{k=0}^{\infty}\left(1-q^{k} x\right) \text { and }(x ; q)_{n}=(x ; q)_{\infty} /\left(q^{n} x ; q\right)_{\infty} .
$$

The goal of this paper will be threefold. Firstly, we shall present proofs (different from Yee's ones) for both identities proposed by Andrews through combinations of the $q$-Gauss summation theorem and Jacobi's triple product identity. Secondly, Andrews' double sum generating functions for partitions with parity index enumerator 
will be reduced to single sum expressions by utilizing the $q$-binomial theorem. Finally, two double sum expansions for $(q x ; q)_{\infty}^{ \pm 1}$ will slightly be generalized with two free variables.

Throughout the paper, the $q$-binomial theorem (cf. [4, II-4]) will be fundamental in our derivation, which reads as

$$
(x ; q)_{n}=\sum_{k=0}^{n}(-1)^{k}\left[\begin{array}{l}
n \\
k
\end{array}\right] q^{\left(\begin{array}{c}
k \\
2
\end{array}\right) x^{k}}
$$

where the $q$-binomial coefficient is defined by

$$
\left[\begin{array}{l}
n \\
k
\end{array}\right]=\frac{(q ; q)_{n}}{(q ; q)_{k}(q ; q)_{n-k}} \text { for } n, k \in \mathbb{N}_{0} \quad \text { with } n \geq k .
$$

\section{SOlutions FOR AndRews' Problems 9 AND 10}

This section will be devoted to the confirmation to the two identities demanded by Andrews [1], which will be accomplished by combining limiting cases of the $q$-Gauss summation theorem with Jacobi's triple product identity. Our proofs are different from those given by Yee [5], who employed a partial theta series identity due to Ramanujan [2, Entry 1.6.2].

Theorem 1 (Andrews, Problem 9 in [1]).

$$
\sum_{n \geq 1}(-1)^{n} q^{n^{2}}=\sum_{i, j \geq 0} \frac{(-1)^{i} q^{(i-j)^{2}+j^{2}+i-j}}{(-q ; q)_{i}(q ; q)_{2 j-1}(q ; q)_{i-2 j+1}} .
$$

Theorem 2 (Andrews, Problem 10 in [1]).

$$
\sum_{n \geq 0}(-1)^{n} q^{n^{2}}=\sum_{i, j \geq 0} \frac{(-1)^{i} q^{(i-j)^{2}+j^{2}+j}}{(-q ; q)_{i}(q ; q)_{2 j}(q ; q)_{i-2 j}} .
$$

Replacing $i$ by $2 j+k-1$, we can express the double sum in (1.1) as

$$
\begin{aligned}
\operatorname{RHS}(1.1) & =\sum_{j \geq 1} \sum_{i \geq 2 j-1} \frac{(-1)^{i} q^{(i-j)^{2}+j^{2}+i-j}}{(-q ; q)_{i}(q ; q)_{2 j-1}(q ; q)_{i-2 j+1}} \\
& =\sum_{j \geq 1} \frac{-q^{2 j^{2}-j}}{\left(q^{2} ; q^{2}\right)_{2 j-1}} \sum_{k \geq 0} \frac{(-1)^{k} q^{k^{2}+2 k j-k}}{(q ; q)_{k}\left(-q^{2 j} ; q\right)_{k}} .
\end{aligned}
$$

The inner sum with respect to $k$ can be evaluated, by the $q$-Gauss summation theorem (cf. $[3, \S 8.4]$ and $\left[4\right.$, II-8]) for ${ }_{2} \phi_{1}$-series, as $\left(-q^{2 j} ; q\right)_{\infty}^{-1}$, which leads the double sum to the following closed form

$$
\operatorname{RHS}(1.1)=\frac{-1}{(-q ; q)_{\infty}} \sum_{j \geq 1} \frac{q^{2 j^{2}-j}}{(q ; q)_{2 j-1}}=\frac{(q ; q)_{\infty}-(-q ; q)_{\infty}}{2(-q ; q)_{\infty}}
$$


where the second $q$-exponential function (cf. [3, §8.2] and [4, II-2]) has been appealed. Finally applying Jacobi's triple product identity (cf. [3, §8.6] and [4, II-28]), we can make further reformulation

$$
\begin{aligned}
2 \operatorname{RHS}(1.1) & =\frac{(q ; q)_{\infty}}{(-q ; q)_{\infty}}-1=\left(q^{2} ; q^{2}\right)_{\infty}\left(q ; q^{2}\right)_{\infty}^{2}-1 \\
& =\sum_{n \in \mathbb{Z}}(-1)^{n} q^{n^{2}}-1=2 \sum_{n \geq 1}(-1)^{n} q^{n^{2}}=2 \operatorname{LHS}(1.1)
\end{aligned}
$$

which proves the first identity displayed in (1.1).

The identity displayed (1.2) can be confirmed analogously. In fact, performing the replacement $i \rightarrow 2 j+k$, we can express the double sum in (1.2) as

$$
\begin{aligned}
\operatorname{RHS}(1.2) & =\sum_{j \geq 0} \sum_{i \geq 2 j} \frac{(-1)^{i} q^{(i-j)^{2}+j^{2}+j}}{(-q ; q)_{i}(q ; q)_{2 j}(q ; q)_{i-2 j}} \\
& =\sum_{j \geq 0} \frac{q^{2 j^{2}+j}}{\left(q^{2} ; q^{2}\right)_{2 j}} \sum_{k \geq 0} \frac{(-1)^{k} q^{k^{2}+2 k j}}{(q ; q)_{k}\left(-q^{2 j+1} ; q\right)_{k}} .
\end{aligned}
$$

By invoking again the $q$-Gauss summation theorem and the second $q$-exponential function, we can proceed with the following simplifications

$$
\operatorname{RHS}(1.2)=\frac{1}{(-q ; q)_{\infty}} \sum_{j \geq 0} \frac{q^{2 j^{2}+j}}{(q ; q)_{2 j}}=\frac{(q ; q)_{\infty}+(-q ; q)_{\infty}}{2(-q ; q)_{\infty}} .
$$

Then the identity (1.2) follows for the same reason as that for (1.1).

\section{Single SUM EXPRESSIONS FOR COROLLARIES 11 AND 13}

For a partition $\lambda=\left(\lambda_{1} \geq \lambda_{2} \geq \cdots \geq \lambda_{\ell}>0\right)$, define its upper even (odd) index by the number of terms in the longest decreasing subsequence of parts beginning with an even (odd) part and alternating in parity. Following Andrews [1], denote by $D_{e}(\infty)$ (resp. $D_{o}(\infty)$ ) the generating function of the partitions into distinct parts in three variables $q, x, y$, which account respectively for weights, lengths and upper even (resp. odd) parity indices. Andrews finds the following double sum expressions.

Lemma 1 (Andrews, Corollary 11 in [1]).

$$
\begin{aligned}
D_{o}(\infty)= & \sum_{i, j \geq 0} \frac{x^{i} y^{2 j} q^{(i-j)^{2}+j^{2}+i+j}}{(-q ; q)_{i}(q ; q)_{2 j}(q ; q)_{i-2 j}} \\
& +\sum_{i, j \geq 0} \frac{x^{i} y^{2 j-1} q^{(i-j)^{2}+j^{2}+i-j}}{(-q ; q)_{i}(q ; q)_{2 j-1}(q ; q)_{i-2 j+1}}
\end{aligned}
$$




$$
\begin{aligned}
D_{e}(\infty)= & \sum_{i, j \geq 0} \frac{x^{i} y^{2 j} q^{(i-j)^{2}+j^{2}+j}}{(-q ; q)_{i}(q ; q)_{2 j}(q ; q)_{i-2 j}} \\
& +\sum_{i, j \geq 0} \frac{x^{i} y^{2 j-1} q^{(i-j)^{2}+j^{2}+2 i-j}}{(-q ; q)_{i}(q ; q)_{2 j-1}(q ; q)_{i-2 j+1}}
\end{aligned}
$$

Recalling the $q$-binomial theorem, we can express $D_{o}(\infty)$ in $(2.1 \mathrm{a}-2.1 \mathrm{~b})$ as follows:

$$
\begin{aligned}
D_{o}(\infty)= & \sum_{i \geq 0} \frac{x^{i} q^{i^{2}+i}}{\left(q^{2} ; q^{2}\right)_{i}} \sum_{j}\left[\begin{array}{c}
i \\
2 j
\end{array}\right] q^{\left(\begin{array}{c}
2 j \\
2
\end{array}\right)+2 j(1-i)} y^{2 j} \\
& +\sum_{i \geq 1} \frac{x^{i} q^{i^{2}}}{\left(q^{2} ; q^{2}\right)_{i}} \sum_{j}\left[\begin{array}{c}
i \\
2 j-1
\end{array}\right] q^{(2 j-1)+(2 j-1)(1-i)} y^{2 j-1} \\
= & \sum_{i \geq 0} \frac{x^{i} q^{i^{2}+i}}{2\left(q^{2} ; q^{2}\right)_{i}}\left\{\left(-q^{1-i} y ; q\right)_{i}+\left(q^{1-i} y ; q\right)_{i}\right\} \\
& +\sum_{i \geq 1} \frac{x^{i} q^{i^{2}}}{2\left(q^{2} ; q^{2}\right)_{i}}\left\{\left(-q^{1-i} y ; q\right)_{i}-\left(q^{1-i} y ; q\right)_{i}\right\}
\end{aligned}
$$

The generating function $D_{e}(\infty)$ in $(2.1 \mathrm{c}-2.1 \mathrm{~d})$ can similarly be treated as follows:

$$
\begin{aligned}
D_{e}(\infty)= & \sum_{i \geq 0} \frac{x^{i} q^{i^{2}}}{\left(q^{2} ; q^{2}\right)_{i}} \sum_{j}\left[\begin{array}{c}
i \\
2 j
\end{array}\right] q^{\left(\begin{array}{c}
2 j \\
2
\end{array}\right)+2 j(1-i)} y^{2 j} \\
& +\sum_{i \geq 1} \frac{x^{i} q^{i^{2}+i}}{\left(q^{2} ; q^{2}\right)_{i}} \sum_{j}\left[\begin{array}{c}
i \\
2 j+1
\end{array}\right] q^{\left(2^{2 j+1}\right)+(2 j+1)(1-i)} y^{2 j+1} \\
= & \sum_{i \geq 0} \frac{x^{i} q^{i^{2}}}{2\left(q^{2} ; q^{2}\right)_{i}}\left\{\left(-q^{1-i} y ; q\right)_{i}+\left(q^{1-i} y ; q\right)_{i}\right\} \\
& +\sum_{i \geq 1} \frac{x^{i} q^{i^{2}+i}}{2\left(q^{2} ; q^{2}\right)_{i}}\left\{\left(-q^{1-i} y ; q\right)_{i}-\left(q^{1-i} y ; q\right)_{i}\right\} .
\end{aligned}
$$

Recombining the summation terms and then inverting the shifted factorials involving $y$, we may state the resulting equations as the following theorem.

Theorem 3 (Single sum generating function).

$$
D_{o}(\infty)=\sum_{i \geq 0} \frac{\left(1+q^{i}\right)(-1 / y ; q)_{i}}{2\left(q^{2} ; q^{2}\right)_{i}} q^{\left(\begin{array}{c}
i \\
2
\end{array}\right)+i}(x y)^{i}
$$




$$
\begin{aligned}
& -\sum_{i \geq 0} \frac{\left(1-q^{i}\right)(1 / y ; q)_{i}}{2\left(q^{2} ; q^{2}\right)_{i}} q^{\left(\begin{array}{c}
i \\
2
\end{array}\right)+i}(-x y)^{i}, \\
D_{e}(\infty)= & \sum_{i \geq 0} \frac{\left(1+q^{i}\right)(-1 / y ; q)_{i}}{2\left(q^{2} ; q^{2}\right)_{i}} q^{\left(\begin{array}{c}
i \\
2
\end{array}\right)+i}(x y)^{i} \\
& +\sum_{i \geq 0} \frac{\left(1-q^{i}\right)(1 / y ; q)_{i}}{2\left(q^{2} ; q^{2}\right)_{i}} q^{\left(\begin{array}{c}
i \\
2
\end{array}\right)+i}(-x y)^{i} .
\end{aligned}
$$

Instead, let $F_{e}(\infty)$ (resp. $\left.F_{o}(\infty)\right)$ be the generating function of the unrestricted partitions in three variables $q, x, y$, which account, respectively, for weights, lengths and upper even (resp. odd) parity indices. Andrews obtains also the following double sum expressions.

Lemma 2 (Andrews, Corollary 13 in [1]).

$$
\begin{aligned}
F_{e}(\infty)= & \sum_{i, j \geq 0} \frac{x^{i} y^{2 j} q^{2 j^{2}+i-j}}{(-q ; q)_{i}(q ; q)_{2 j}(q ; q)_{i-2 j}} \\
& +\sum_{i, j \geq 0} \frac{x^{i} y^{2 j+1} q^{2 j^{2}+2 i+j}}{(-q ; q)_{i}(q ; q)_{2 j+1}(q ; q)_{i-2 j-1}}, \\
F_{o}(\infty)= & \sum_{i, j \geq 0} \frac{x^{i} y^{2 j} q^{2 j^{2}+2 i-j}}{(-q ; q)_{i}(q ; q)_{2 j}(q ; q)_{i-2 j}} \\
& +\sum_{i, j \geq 0} \frac{x^{i} y^{2 j+1} q^{2 j^{2}+i+j}}{(-q ; q)_{i}(q ; q)_{2 j+1}(q ; q)_{i-2 j-1}} .
\end{aligned}
$$

Following exactly the same procedure, we can show that both $F_{e}(\infty)$ and $F_{o}(\infty)$ can be reduced to the following single sum expressions.

Theorem 4 (Single sum generating function).

$$
\begin{aligned}
& F_{e}(\infty)=\sum_{i \geq 0} \frac{\left(1+q^{i}\right)(-y ; q)_{i}}{2\left(q^{2} ; q^{2}\right)_{i}}(q x)^{i}+\sum_{i \geq 0} \frac{\left(1-q^{i}\right)(y ; q)_{i}}{2\left(q^{2} ; q^{2}\right)_{i}}(q x)^{i}, \\
& F_{o}(\infty)=\sum_{i \geq 0} \frac{\left(1+q^{i}\right)(-y ; q)_{i}}{2\left(q^{2} ; q^{2}\right)_{i}}(q x)^{i}-\sum_{i \geq 0} \frac{\left(1-q^{i}\right)(y ; q)_{i}}{2\left(q^{2} ; q^{2}\right)_{i}}(q x)^{i}
\end{aligned}
$$

\section{TWO PARAMETER EXTENSIONS OF EQUATIONS (9.18) AND (10.19)}

Letting $y=1$ in Lemmas 1 and 2, Andrews deduced, in view of partition interpretations, the following two interesting identities. 
Proposition 1 (Andrews, Equation 9.18 in [1]).

$$
\begin{aligned}
(-q x ; q)_{\infty}= & \sum_{i, j \geq 0} \frac{x^{i} q^{(i-j)^{2}+j^{2}+i+j}}{(-q ; q)_{i}(q ; q)_{2 j}(q ; q)_{i-2 j}} \\
& +\sum_{i, j \geq 0} \frac{x^{i} q^{(i-j)^{2}+j^{2}+i-j}}{(-q ; q)_{i}(q ; q)_{2 j-1}(q ; q)_{i-2 j+1}} \\
= & \sum_{i, j \geq 0} \frac{x^{i} q^{(i-j)^{2}+j^{2}+j}}{(-q ; q)_{i}(q ; q)_{2 j}(q ; q)_{i-2 j}} \\
& +\sum_{i, j \geq 0} \frac{x^{i} q^{(i-j)^{2}+j^{2}+3 j+1}}{(-q ; q)_{i}(q ; q)_{2 j+1}(q ; q)_{i-2 j-1}} .
\end{aligned}
$$

Proposition 2 (Andrews, Equation 10.19 in [1]).

$$
\begin{aligned}
\frac{1}{(q x ; q)_{\infty}}= & \sum_{i, j \geq 0} \frac{x^{i} q^{2 j^{2}+i-j}}{(-q ; q)_{i}(q ; q)_{2 j}(q ; q)_{i-2 j}} \\
& +\sum_{i, j \geq 0} \frac{x^{i} q^{2 j^{2}+2 i+j}}{(-q ; q)_{i}(q ; q)_{2 j+1}(q ; q)_{i-2 j-1}} \\
= & \sum_{i, j \geq 0} \frac{x^{i} q^{2 j^{2}+2 i-j}}{(-q ; q)_{i}(q ; q)_{2 j}(q ; q)_{i-2 j}} \\
& +\sum_{i, j \geq 0} \frac{x^{i} q^{2 j}+i+j}{(-q ; q)_{i}(q ; q)_{2 j+1}(q ; q)_{i-2 j-1}} .
\end{aligned}
$$

However, if we relate Lemmas 1 and 2 respectively to Theorems 3 and 4, the last two identities follow immediately from the first and the second $q$-exponential functions. This observation suggests that there may exist more general identities behind these two examples. In fact, this can be realized by examining the following nonterminating $q$-binomial series (cf. [3, §8.1] and [4, II-3]):

$$
\frac{(q x ; q)_{\infty}}{(q y ; q)_{\infty}}=\sum_{k \geq 0} \frac{(x / y ; q)_{k}}{(q ; q)_{k}}(q y)^{k} \quad \text { where }|q y|<1
$$

Observe the two almost trivial equations

$$
1=\frac{\left(-q^{1-i} ; q\right)_{i}+\left(q^{1-i} ; q\right)_{i}}{\left(-q^{1-i} ; q\right)_{i}}=\frac{1+q^{i}}{(-q ; q)} q^{\left(\begin{array}{l}
i \\
2
\end{array}\right)} \sum_{j}\left[\begin{array}{c}
i \\
2 j
\end{array}\right] q^{2 j^{2}-2 i j+j}, \quad i>0 ;
$$




$$
1=\frac{\left(-q^{1-i} ; q\right)_{i}-\left(q^{1-i} ; q\right)_{i}}{\left(-q^{1-i} ; q\right)_{i}}=\frac{1+q^{i}}{(-q ; q)_{i}} q^{\left(\begin{array}{c}
i \\
2
\end{array}\right)} \sum_{j}\left[\begin{array}{c}
i \\
2 j-1
\end{array}\right] q^{2 j^{2}-2 i j+i-j}, \quad i>0 .
$$

We can manipulate the $q$-binomial sum as follows

$$
\begin{aligned}
\frac{(q x ; q)_{\infty}}{(q y ; q)_{\infty}}= & \sum_{i \geq 0} \frac{(x / y ; q)_{i}}{(q ; q)_{i}}(q y)^{i} \frac{1+q^{i}}{1+q^{i}} \\
= & \sum_{i \geq 0} \frac{(x / y ; q)_{i}}{(q ; q)_{i}} \frac{(q y)^{i}}{1+q^{i}} \frac{\left(-q^{1-i} ; q\right)_{i}+\left(q^{1-i} ; q\right)_{i}}{\left(-q^{1-i} ; q\right)_{i}} \\
& +\sum_{i \geq 0} \frac{(x / y ; q)_{i}}{(q ; q)_{i}} \frac{\left(q^{2} y\right)^{i}}{1+q^{i}} \frac{\left(-q^{1-i} ; q\right)_{i}-\left(q^{1-i} ; q\right)_{i}}{\left(-q^{1-i} ; q\right)_{i}} \\
= & \sum_{i \geq 0} \frac{(x / y ; q)_{i}}{(q ; q)_{i}} \frac{(q y)^{i}}{(-q ; q)_{i}} \sum_{j}\left[\begin{array}{c}
i \\
2 j
\end{array}\right] q^{\left(\begin{array}{c}
i \\
2
\end{array}\right)+2 j^{2}-2 i j+j} \\
& +\sum_{i \geq 0} \frac{(x / y ; q)_{i}}{(q ; q)_{i}} \frac{\left(q^{2} y\right)^{i}}{(-q ; q)_{i}} \sum_{j}\left[\begin{array}{c}
i \\
2 j-1
\end{array}\right] q^{\left(\begin{array}{c}
i \\
2
\end{array}\right)+2 j^{2}-2 i j+i-j}
\end{aligned}
$$

By switching the two terms in the linear factor $1+q^{i}$, we may alternatively reformulate the $q$-binomial sum as

$$
\begin{aligned}
\frac{(q x ; q)_{\infty}}{(q y ; q)_{\infty}}= & \sum_{i \geq 0} \frac{(x / y ; q)_{i}}{(q ; q)_{i}}(q y)^{i} \frac{1+q^{i}}{1+q^{i}} \\
= & \sum_{i \geq 0} \frac{(x / y ; q)_{i}}{(q ; q)_{i}} \frac{\left(q^{2} y\right)^{i}}{1+q^{i}} \frac{\left(-q^{1-i} ; q\right)_{i}+\left(q^{1-i} ; q\right)_{i}}{\left(-q^{1-i} ; q\right)_{i}} \\
& +\sum_{i \geq 0} \frac{(x / y ; q)_{i}}{(q ; q)_{i}} \frac{(q y)^{i}}{1+q^{i}} \frac{\left(-q^{1-i} ; q\right)_{i}-\left(q^{1-i} ; q\right)_{i}}{\left(-q^{1-i} ; q\right)_{i}} \\
= & \sum_{i \geq 0} \frac{(x / y ; q)_{i}}{(q ; q)_{i}} \frac{\left(q^{2} y\right)^{i}}{(-q ; q)_{i}} \sum_{j}\left[\begin{array}{c}
i \\
2 j
\end{array}\right] q^{\left(\begin{array}{c}
i \\
2
\end{array}\right)+2 j^{2}-2 i j+j} \\
& +\sum_{i \geq 0} \frac{(x / y ; q)_{i}}{(q ; q)_{i}} \frac{(q y)^{i}}{(-q ; q)_{i}} \sum_{j}\left[\begin{array}{c}
i \\
2 j-1
\end{array}\right] q^{\left(\begin{array}{c}
i \\
2
\end{array}\right)+2 j^{2}-2 i j+i-j}
\end{aligned}
$$

Writing the Gaussian binomial coefficients in terms of shifted factorials, we get the following two bivariate series identities. 
Theorem 5 (Two parameter generalization of Proposition 1).

$$
\begin{aligned}
\frac{(q x ; q)_{\infty}}{(q y ; q)_{\infty}}= & \sum_{i, j \geq 0} \frac{y^{i}(x / y ; q)_{i} q^{\left(\begin{array}{c}
i \\
2
\end{array}\right)+2 j^{2}-2 i j+i+j}}{(-q ; q)_{i}(q ; q)_{2 j}(q ; q)_{i-2 j}} \\
& +\sum_{i, j \geq 0} \frac{y^{i}(x / y ; q)_{i} q^{\left(\begin{array}{c}
i \\
2
\end{array}\right)+2 j^{2}-2 i j+3 i-j}}{(-q ; q)_{i}(q ; q)_{2 j-1}(q ; q)_{i-2 j+1}} \\
\frac{(q x ; q)_{\infty}}{(q y ; q)_{\infty}}= & \sum_{i, j \geq 0} \frac{y^{i}(x / y ; q)_{i} q^{\left(\begin{array}{l}
i \\
2
\end{array}\right)+2 j^{2}-2 i j+2 i+j}}{(-q ; q)_{i}(q ; q)_{2 j}(q ; q)_{i-2 j}} \\
& +\sum_{i, j \geq 0} \frac{y^{i}(x / y ; q)_{i} q^{\left(\begin{array}{c}
i \\
2
\end{array}\right)+2 j^{2}-2 i j+2 i-j}}{(-q ; q)_{i}(q ; q)_{2 j-1}(q ; q)_{i-2 j+1}}
\end{aligned}
$$

When $y \rightarrow 0$, this theorem gives the identities displayed in Proposition 1 . Similarly, consider two further simple equations

$$
\begin{aligned}
& 1=\frac{(-1 ; q)_{i}+(1 ; q)_{i}}{(-1 ; q)_{i}}=\frac{1+q^{i}}{(-q ; q)_{i}} \sum_{j}\left[\begin{array}{c}
i \\
2 j
\end{array}\right] q^{2 j^{2}-j}, \quad i>0 ; \\
& 1=\frac{(-1 ; q)_{i}-(1 ; q)_{i}}{(-1 ; q)_{i}}=\frac{1+q^{i}}{(-q ; q)_{i}} \sum_{j}\left[\begin{array}{c}
i \\
2 j+1
\end{array}\right] q^{2 j^{2}+j}, \quad i>0 .
\end{aligned}
$$

We can express the $q$-binomial sum as follows

$$
\begin{aligned}
\frac{(q x ; q)_{\infty}}{(q y ; q)_{\infty}}= & \sum_{i \geq 0} \frac{(x / y ; q)_{i}}{(q ; q)_{i}}(q y)^{i} \frac{1+q^{i}}{1+q^{i}} \\
= & \sum_{i \geq 0} \frac{(x / y ; q)_{i}}{(q ; q)_{i}} \frac{(q y)^{i}}{1+q^{i}} \frac{(-1 ; q)_{i}+(1 ; q)_{i}}{(-1 ; q)_{i}} \\
& +\sum_{i \geq 0} \frac{(x / y ; q)_{i}}{(q ; q)_{i}} \frac{\left(q^{2} y\right)^{i}}{1+q^{i}} \frac{(-1 ; q)_{i}-(1 ; q)_{i}}{(-1 ; q)_{i}} \\
= & \sum_{i \geq 0} \frac{(x / y ; q)_{i}}{(q ; q)_{i}} \frac{(q y)^{i}}{(-q ; q)_{i}} \sum_{j}\left[\begin{array}{c}
i \\
2 j
\end{array}\right] q^{2 j^{2}-j} \\
& +\sum_{i \geq 0} \frac{(x / y ; q)_{i}}{(q ; q)_{i}} \frac{\left(q^{2} y\right)^{i}}{(-q ; q)_{i}} \sum_{j}\left[\begin{array}{c}
i \\
2 j
\end{array}\right] q^{2 j^{2}+j}
\end{aligned}
$$


By interchanging the two terms in the linear factor $1+q^{i}$, we may alternatively reformulate the $q$-binomial sum as

$$
\begin{aligned}
\frac{(q x ; q)_{\infty}}{(q y ; q)_{\infty}}= & \sum_{i \geq 0} \frac{(x / y ; q)_{i}}{(q ; q)_{i}}(q y)^{i} \frac{1+q^{i}}{1+q^{i}} \\
= & \sum_{i \geq 0} \frac{(x / y ; q)_{i}}{(q ; q)_{i}} \frac{\left(q^{2} y\right)^{i}}{1+q^{i}} \frac{(-1 ; q)_{i}+(1 ; q)_{i}}{(-1 ; q)_{i}} \\
& +\sum_{i \geq 0} \frac{(x / y ; q)_{i}}{(q ; q)_{i}} \frac{(q y)^{i}}{1+q^{i}} \frac{(-1 ; q)_{i}-(1 ; q)_{i}}{(-1 ; q)_{i}} \\
= & \sum_{i \geq 0} \frac{(x / y ; q)_{i}}{(q ; q)_{i}} \frac{\left(q^{2} y\right)^{i}}{(-q ; q)_{i}} \sum_{j}\left[\begin{array}{c}
i \\
2 j
\end{array}\right] q^{2 j^{2}-j} \\
& +\sum_{i \geq 0} \frac{(x / y ; q)_{i}}{(q ; q)_{i}} \frac{(q y)^{i}}{(-q ; q)_{i}} \sum_{j}[2 j+1] q^{2 j^{2}+j} .
\end{aligned}
$$

Writing the Gaussian binomial coefficients in terms of shifted factorials, we get the following two bivariate series identities.

Theorem 6 (Two parameter generalization of Proposition 2).

$$
\begin{aligned}
\frac{(q x ; q)_{\infty}}{(q y ; q)_{\infty}}= & \sum_{i, j \geq 0} \frac{y^{i}(x / y ; q)_{i} q^{2 j^{2}+i-j}}{(-q ; q)_{i}(q ; q)_{2 j}(q ; q)_{i-2 j}} \\
& +\sum_{i, j \geq 0} \frac{y^{i}(x / y ; q)_{i} q^{2 j^{2}+2 i+j}}{(-q ; q)_{i}(q ; q)_{2 j+1}(q ; q)_{i-2 j-1}} \\
\frac{(q x ; q)_{\infty}}{(q y ; q)_{\infty}}= & \sum_{i, j \geq 0} \frac{y^{i}(x / y ; q)_{i} q^{2 j^{2}+2 i-j}}{(-q ; q)_{i}(q ; q)_{2 j}(q ; q)_{i-2 j}} \\
& +\sum_{i, j \geq 0} \frac{y^{i}(x / y ; q)_{i} q^{2 j^{2}+i+j}}{(-q ; q)_{i}(q ; q)_{2 j+1}(q ; q)_{i-2 j-1}}
\end{aligned}
$$

When $x=0$, this theorem gives the identities displayed in Proposition 2.

Open Problem: It would be interesting to find combinatorial interpretations via partitions for the two variable identities displayed in Theorems 9 and 10.

\section{REFERENCES}

[1] G. E. Andrews, "Parity in partition identities," Ramanujan J., vol. 23, no. 1-3, pp. 45-90, 2010.

[2] G. E. Andrews and B. C. Berndt, Ramanujan's lost notebook. Part II. New York: Springer, 2009. 
[3] W. N. Bailey, Generalized hypergeometric series, ser. Cambridge Tracts in Mathematics and Mathematical Physics. London: Cambridge University Press, 1935, vol. 32.

[4] G. Gasper and M. Rahman, Basic hypergeometric series. 2nd ed., ser. Encyclopedia of Mathematics and Its Applications. Cambridge: Cambridge University Press, 2004, vol. 96.

[5] A. J. Yee, "Ramanujan's partial theta series and parity in partitions," Ramanujan J., vol. 23, no. 1-3, pp. $215-225,2010$.

Author's address

\section{Wenchang Chu}

Dipartimento di Matematica e Fisica "Ennio De Giorgi”, Universit del Salento, Lecce-Arnesano P.

O. Box 193, 73100 Lecce, Italy

E-mail address: chu. wenchang@unisalento. it 